\title{
The property transmission hypothesis: A possible explanation for visual impressions of pulling and other kinds of phenomenal causality
}

\author{
Peter A White \\ School of Psychology, Cardiff University, Tower Building, Park Place, Cardiff CF10 3YG, Wales, UK; \\ e-mail:whitepa@cardiff.ac.uk
}

Received 28 August 2009, in revised form 6 July 2010; published online 25 August 2010

\begin{abstract}
Under certain circumstances, stimuli involving two moving objects that do not come into contact reliably give rise to the illusory perceptual impression that one of the objects is pulling the other, as if there is an unseen connection between them. It is proposed that the conditions determining the occurrence of this impression can be explained as cases of application of the property-transmission hypothesis. This is a general hypothesis that causal objects operate in part by transmitting some of their own properties to effect objects under conditions where the causal object is active, where there are cues to the occurrence of generative transmission between the causal object and an effect object, and where there is a time-ordered relation of resemblance between properties of the causal object and those of the effect object. This hypothesis predicts that the pulling impression should occur only when the effect object adopts kinematic properties (speed and direction) that resemble those of the causal object. An experiment is reported that supports this prediction.
\end{abstract}

\section{Introduction}

Simple animations depicting geometrical shapes moving against a uniform background can give rise to perceptual impressions of causality. One object can be perceived as causing some kind of outcome for another object, even though no causal relation is present in the stimuli. This was first documented by Michotte (1963), and a substantial body of research since then has confirmed Michotte's findings, confirmed that the impressions are part of the visual perception of the stimuli, and also demonstrated the occurrence of qualitatively different visual impressions of causality with a diverse array of stimuli (Choi and Scholl 2004, 2006; Gordon et al 1990; Natsoulas 1961; Newman et al 2008; Saxe and Carey 2006; Schlottmann et al 2006; Scholl and Tremoulet 2000; White and Milne 1997, 1999, 2003).

In a typical stimulus for one kind of causal impression, two similar objects are visible, usually opaque rectangles or discs. One (object B) is stationary in the centre of the screen. The other (object A) enters from the side of the screen, moving horizontally at constant speed. When it contacts object B, object A stops moving and object B starts moving in the same direction and at the same or slightly lesser speed. Observers consistently report an impression that object A makes object B move, an impression known in English as the "launching effect" (Michotte 1963), which is a translation of the original term, "l'effet lancement" (Michotte 1946). Numerous studies have shown that most adult observers report the occurrence of the launching effect when shown stimuli of the kind described, and infants a few months old respond as if they have similar perceptions (Gordon et al 1990; Leslie and Keeble 1987; Newman et al 2008; Saxe and Carey 2006; Schlottmann et al 2006; Scholl and Tremoulet 2000).

The main aim of this paper is to test a novel hypothesis about visual causal impressions. In essence, the hypothesis is that visual impressions of causality tend to occur when the kinematic properties of one object are perceptibly acquired by another object under conditions where there are cues to the possible occurrence of an interaction between the two objects. I shall introduce this hypothesis, show how it applies 
to the two fundamental kinds of causal impression investigated by Michotte (1963), launching and entraining, and then report a test of the hypothesis using stimuli that tend to give rise to a qualitatively different causal impression in which one object is perceived as pulling one or more other objects (Michotte 1963; White and Milne 1997).

I have proposed that many kinds of causal perception and inference are influenced by a general hypothesis that properties of causal objects tend to be transmitted to effect objects (White 2009). Numerous examples in different domains show that property transmission is a common and readily encountered occurrence. Leaving aside launching, entraining, and pulling, it occurs in many kinds of interactions between objects, such as staining or marking events where colour in one object (eg a pen or paintbrush) is transmitted to another object, in interactions between relatively rigid and relatively yielding objects (eg shoes and mud, which can retain shoeprints), in other kinds of processes such as heating, cooling, and making wet, in biological processes such as reproduction (cats produce cats and not elm trees), and in the psychological domain (eg the content of an action resembles the content of an intention that is believed to give rise to the action).

I proposed that the conjunction of three criteria serves to identify perceived or judged property transmission. One is that there must be some kind of perceived or described activity going on. This is based on an argument that activity is important to the differentiation between causal and effect objects in interactions such as collision events (White 2006). Thus, two similar chairs sitting motionless side-by-side do not meet the activity criterion. Another is that there must be perceived or described cues to generative transmission from one object to another. These are observable cues to the transmission of causally efficacious force or energy from one object to another, either directly or through a medium. An example would be a gust of wind between a bellows and a candle flame, which functions as a cue to a causal relation in which the bellows extinguishes the flame. This is based on research by Shultz (1982) showing that generative transmission cues play an important role in causal inference at least from the age of two years, and are preferred to other kinds of cues to causal inference. As the bellows example shows, physical contact is not necessary, so long as there is some cue to the occurrence of transmission through an intervening medium. Thus, one chair on wheels rolling past another without touching it does not meet the generative transmission criterion (though it might, if it produced a strong enough gust of wind to move the other chair). The third criterion is that the interaction should be characterised by a salient time-ordered resemblance between the properties of causal object and effect object. Specifically, there should be a resemblance between some property of the causal object prior to the interaction and some novel property of the effect object present after the interaction. Thus, if one chair is seen to bump into another, this will not be interpreted as the cause of any of the second chair's preexisting properties, even if the two chairs are visibly identical. These criteria distinguish similarity due to causal interactions involving property transmission from similarity due to common generative processes, such as the manufacturing process that constructed the two chairs, and from accidental similarity, such as a potato that resembles a human face.

White (2009) argued that the property-transmission hypothesis can explain the occurrence of perceptual impressions of causality. That argument was applied mainly to the launching effect. The typical stimulus for the launching effect meets the three criteria for ascription of property transmission. The causal object (A) is active, in motion; there are cues to the occurrence of generative transmission, involving object A's approach to and contact with object B; and an immediate outcome of the interaction is that object $\mathrm{B}$ acquires novel kinematic properties that resemble those that object A possessed prior to the interaction. This account predicts that the launching 
effect should be maximal or most likely to occur when the kinematic properties of object B immediately after the interaction most closely resemble those of object A prior to the interaction.

Natsoulas (1961) found that this is the case for speed: the launching effect appears strongest when object B's speed after contact is the same as object A's speed prior to contact, and it weakens or disappears as the two speeds diverge. Michotte (1963) reported that it is also the case for direction. The launching effect occurs most strongly or reliably when object B's direction of motion is the same as object A's direction prior to contact, and even quite small divergence in direction (as little as $25^{\circ}$ ) resulted in considerable weakening of the causal impression. Beasley (1968) presented a stimulus in which object B moved off at right angles to object A's direction, and $45 \%$ of responses to this stimulus were classified as causal responses, compared to $64 \%$ for a standard launching stimulus. However, Beasley asked his participants to choose which of two statements they thought would be more correct to describe the impression they saw, and the wording for the causal description was: "The movement of the black square to the right caused the movement of the red square to the right" (page 403). It is not clear that participants would endorse this descriptor only if they had a visual causal impression. The use of the term "caused" in particular is rather abstract, and could lead participants to endorse it, if they judged that the movement of the red square was in some way dependent on the movement of the black square, regardless of what kind of visual impression they had. Further investigation of the effect of direction on the visual impression of causality would be of some value.

The property transmission hypothesis also accounts for the effect of delay on the occurrence of the causal impression. Michotte (1963) found that introducing delay between the moment at which object $\mathrm{A}$ contacted object $\mathrm{B}$ and the moment at which object B started moving had a striking effect on reported visual impressions. With delays up to $56 \mathrm{~ms}$ the launching effect was reported on almost all trials. Increasing the delay to about $98 \mathrm{~ms}$ led to an impression that Michotte called "delayed launching"; the launching effect was still reported, but observers felt that something was holding up object B's motion, as if it was sticking to object A. At longer delays the dominant impression was that object B moved of its own accord. It is important to note a parallel with another experiment by Michotte (1963) in which a single object moved horizontally at constant speed and paused for a brief duration in the middle of its motion. Pauses less than $56 \mathrm{~ms}$ in duration were not perceived. This means that the causal impression begins to change as soon as a noticeable pause in motion occurs. A noticeable pause means that object $\mathrm{A}$ is recognised as not active when object $\mathrm{B}$ starts to move. Thus, the activity criterion is not met and the property-transmission hypothesis predicts that the causal impression will not occur. The intermediate impression of delayed launching could be attributed to subjective uncertainty whether object A was truly inactive before object B began to move, perhaps coupled with uncertainty over whether a pause had occurred.

The typical stimulus for the other kind of causal impression regarded as fundamental by Michotte (1963), entraining, is similar to that for launching except that, at contact, object A continues to move in the same direction and at the same speed, so that it remains in contact with object $\mathrm{B}$. This results in an impression that object $\mathrm{A}$ is pushing or carrying object $\mathrm{B}$. In this case too, object $\mathrm{B}$ acquires the kinematic properties that object $\mathrm{A}$ has prior to contact at the moment when visible cues indicate the occurrence of an interaction. There has been little further research on entraining so it is not clear what effect variations in variables such as relative speeds before and after contact would have. However, the typical stimulus used by Michotte exactly fits the conditions under which property transmission should be perceived. 
To the extent that the property-transmission hypothesis is a general feature of causal perception, it is not specific to the launching and entraining effects and should hold for other kinds of visual causal impression as well. The main aim of the present research, therefore, is to test the predictions of the property-transmission hypothesis for a different kind of visual causal impression, pulling.

Michotte (1963) was the first to report that an impression of pulling occurred with certain stimuli. He reported that it occurred in perception of a stimulus in which a moving object passed by a stationary one, after which the stationary one started moving at the same speed and in the same direction as the initially moving one. The pulling impression was investigated more extensively by White and Milne (1997). The basic stimulus in that research comprised an initial setup of five black rectangles arranged in a column with small but clearly visible gaps between them. The top object began to move from left to right at constant speed. After a short delay, the second object began to move in the same direction at the same speed, and so on until all five rectangles were in motion. Observers of the type stimulus tended to report a strong impression that the first object was pulling the others, despite the absence of any visible connection between them.

White and Milne found that varying the delay between onset of motion of successive objects had little effect on the reported impression. The speed at which the objects moved (with all objects moving at the same speed) and the direction of motion on the monitor screen (horizontal vs up vs down) had no significant effect. If the objects began to move simultaneously, ratings of pulling were so low as to suggest that no pulling impression occurred. If each object rapidly decelerated to a standstill immediately after beginning to move, so that each object stopped moving before the adjacent object started to move, the impression was again much attenuated. These findings fit with the property-transmission hypothesis. If all objects start moving at the same time then there is no time-ordered resemblance between the properties of causal object and effect object. If one object stops moving before the next object starts moving, the activity criterion is not met, because no object is active prior to the onset of motion in the object in question. The property-transmission hypothesis therefore predicts the low pulling ratings obtained under those conditions.

The most important test of the property-transmission hypothesis, as in the case of launching, concerns effects of relative speed and direction. These variables were not manipulated in the study by White and Milne (1997), and they are the main focus of the present research. The stimuli in the present research show just two objects rather than five, and I shall write in terms of two objects from here on, with object A designating the object that starts moving first and object $\mathrm{B}$ designating the other object. The type of stimulus used in this research is illustrated in figure 1 . Figure 1 presents actual frames from the experimental stimuli, reduced in scale and with arrows added to indicate directions of motion. Figure la shows the first frame of the stimulus, in which both objects are stationary. After a brief pause the top object (object A) moves off to the right as shown by the arrow in figure $1 \mathrm{~b}$. Figure $1 \mathrm{~b}$ shows the last frame in which the lower object (object B) is stationary. Then B begins to move in the same direction and with the same speed as A. Figure 1c shows a frame from later in the stimulus when both objects are in motion. Motion continues until both objects exit the frame.

For object A to be perceived as pulling object B, the property-transmission hypothesis predicts that time-ordered resemblance in the kinematic properties of the objects is required. Thus, a pulling impression should be reported if both objects move in the same direction and at the same speed, and a pulling impression should not be reported if the objects move in different directions or at different speeds. The experiment was designed to test these predictions. 


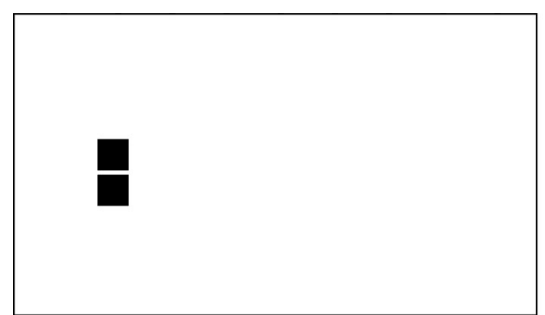

(a)

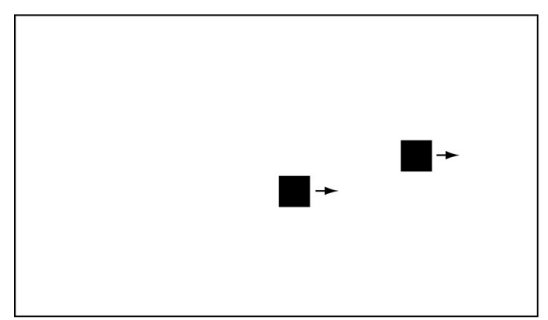

(c)

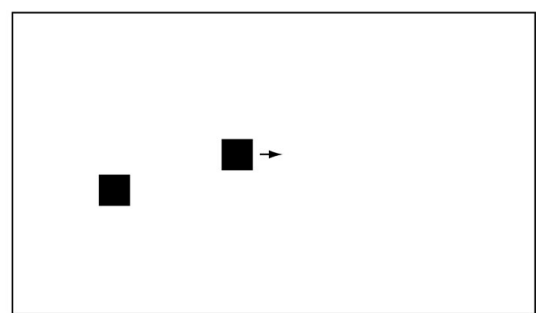

(b)

Figure 1. Example of stimulus sequence. (a) The first frame of the stimulus, with two stationary objects arranged one above the other and separated by a narrow gap. After a fraction of a second, object A (the top object) begins to move to the right. After a further short interval, object B (the lower object) begins to move in the same way. (b) The frame immediately prior to the one in which object B's motion commences. The two objects continue to move across the screen at the same speed and in the same direction. (c) A frame from later in the stimulus. Arrows have been added to show direction of motion.

\section{Method}

\subsection{Participants}

The participants were twenty-nine volunteer undergraduate students, participating in return for cash payment. One participant was excluded prior to analysis because information about the order of presentation of stimuli was lost, so $n=28$ in all analyses.

\subsection{Apparatus, stimulus materials, and design}

Stimuli consisted of frame sequences generated by a Macintosh G3 computer and displayed on a Mitsubishi Diamond Plus 71 16-inch CRT colour monitor. The frames were presented in phase with the computer's vertical blank signal and therefore appeared at the refresh rate of $74 \mathrm{~Hz}$. Each frame was 500 pixels $(18.5 \mathrm{~cm})$ wide by 300 pixels $(11.1 \mathrm{~cm})$ high. The boundaries of this frame appeared on the screen as thin black lines. These disappeared between trials, leaving the screen uniform white. To achieve the necessary presentation rate, frames employed monochrome graphics only. All sequences consisted of 200 frames, lasting $2.7 \mathrm{~s}$. The background of each frame was uniform white throughout. In all stimuli there were two objects. Both were black rectangles, each side being $1.1 \mathrm{~cm}$ (30 pixels) in length. Object boundaries were clearly defined and object motion was completely smooth.

Stimuli were variations on the stimulus depicted in figure 1. Five variables were manipulated.

Time between onset of motion of the object was manipulated (called 'delay'), with two levels, 0.27 and $0.54 \mathrm{~s}$ (called 'short' and 'long' respectively).

Speed of A and speed of B were manipulated, each with two levels, $5.4 \mathrm{~cm} \mathrm{~s}^{-1}$ (called 'slow') and $8.1 \mathrm{~cm} \mathrm{~s}^{-1}$ (called 'moderate'). Speed was measured along the axis of motion.

Direction of A and direction of B were manipulated, each with two levels, 'horizontal' and inclined upwards at an angle of $30^{\circ}$ to the horizontal ('oblique'). 
Figure 1 schematically depicts the combination of long delay, moderate speed of both objects, and horizontal direction of both objects. Figure 2 schematically depicts the stimulus with long delay, slow speed of both objects, object A moving horizontally and object B moving obliquely. Figure 2a shows the first frame in which both objects are stationary, as in figure 1 . Object $A$ then begins to move to the right, as in figure 1. Figure $2 \mathrm{~b}$ shows this, again with an arrow to indicate direction of motion. Then object $\mathrm{B}$ begins to move to the right but upwards at an angle of $30^{\circ}$. Figure $2 \mathrm{c}$ shows that this takes object B above object A. Figure $2 d$ shows the situation a little later, shortly before both objects exit the frame.

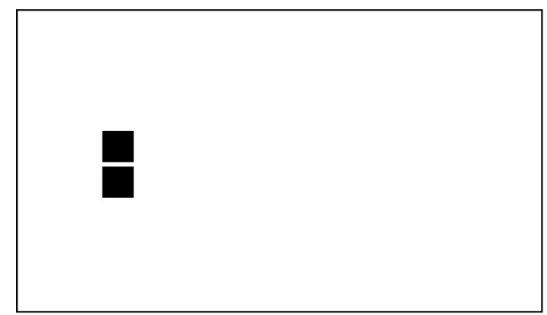

(a)

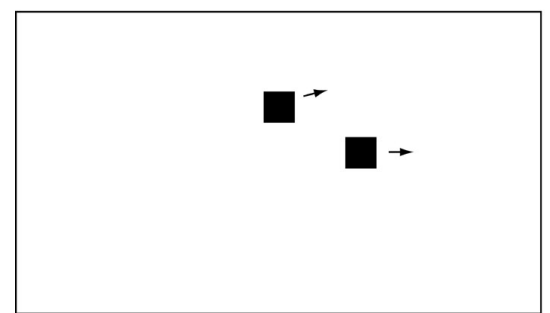

(c)

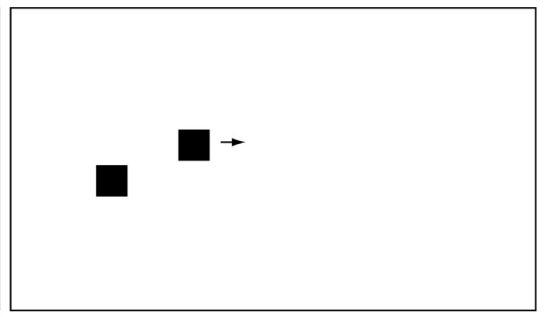

(b)

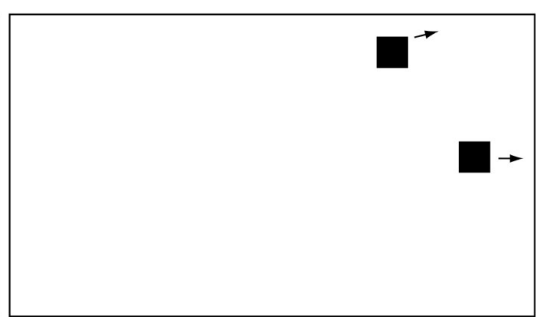

(d)

Figure 2. Stimulus with long delay, slow speed of both objects, object A moving horizontally and object B moving obliquely. (a) The first frame of the stimulus, which is the same as in figure la. (b) The frame immediately prior to the one in which object B's motion commences. (c) and (d) Stages in the progress of both objects across the frame: in both cases object $\mathrm{B}$ is the upper object because its oblique trajectory has taken it above object A. Arrows have been added to show direction of motion.

\subsection{Procedure}

Participants took part in a small laboratory, empty except for the equipment used for the experiment and with fluorescent lighting giving a low ambient light level. Participants were seated so that their faces were approximately $75 \mathrm{~cm}$ from the screen and were permitted to adjust this distance slightly for personal comfort. The experimenter introduced the experiment by giving the participants written instructions informing them that they would be seeing a series of short movies, each involving two objects moving in various different ways. The instructions continued:

"After each movie you will be asked to make a rating of what you have seen. This rating should be made on a scale from 0 to 100 :

0 (zero) means that it seems as though each object starts moving of its own accord.

100 means that it seems as though the object that starts moving first pulls the other one, as if they are connected in some way.

The stronger the impression you have that the first object seems to pull the second one, the higher the number you should put. You can put any number from 0 to 100 .

The experimenter will show you each movie three times. Please make your rating after the third showing. The first two trials will be practice trials. They are a chance for you to get used to the kind of events you will be seeing and the ratings you are asked to make. If you feel you would like more than two practice trials, please say so and you can have as many as you wish. If you have any doubts about how to make your rating, please consult the experimenter." 
The two practice trials were similar to the stimulus shown in figure 1, except that time between onset of motion of the objects was $0.135 \mathrm{~s}$, and in the second practice trial the initial position of object $\mathrm{A}$ was $1.48 \mathrm{~cm}$ to the right of its position in figure la. No participants requested extra practice trials.

The experimental trials then began. Before each trial the experimenter asked if the participant was ready, and participants were instructed to take this as a cue to watch the screen. When they indicated that they were ready, the stimulus was shown three times. Participants were instructed to make their rating after the third showing, but were not prevented from making it sooner. When the participant had made a rating and the stimulus had been shown three times, the experimenter moved on to the next stimulus and repeated the same procedure.

Stimuli were randomly ordered and a different random order was generated for each participant. Each stimulus was rated once, so the total number of stimuli and ratings was $2 \times 2 \times 2 \times 2 \times 2=32$. At the end of the session participants were thanked, paid, and debriefed.

\section{Results}

Ratings were analysed with a 2 (delay: short versus long) $\times 2$ (speed of A: slow versus moderate) $\times 2$ (speed of $\mathrm{B}$ : slow versus moderate) $\times 2$ (direction of A: horizontal versus oblique) $\times 2$ (direction of $\mathrm{B}$ : horizontal versus oblique) analysis of variance (ANOVA) with repeated measures on all five variables. Degrees of freedom are 1 and 27 in all effects. In a five-way ANOVA design there are 31 effects and no predictions were made for most of these. The use of the Bonferroni correction for this number of effects would result in a significant level of 0.002 . On the other hand, less than two effects would be significant at the 0.05 level by chance alone, yet 14 effects were significant at that level (including the effects predicted under the property-transmission hypothesis). Accordingly, a less conservative significance level of 0.01 was adopted. Mean ratings of all stimuli are shown in table 1.

The most important results are three significant interactions that relate to the property-transmission hypothesis. The interactions show that, as predicted, ratings of pulling were high when both objects moved at the same speed and in the same direction, but low when either speed or direction differed between the objects.

Table 1. Mean pulling ratings, all stimuli.

\begin{tabular}{|c|c|c|c|c|c|}
\hline \multicolumn{2}{|l|}{ Speed } & \multicolumn{2}{|l|}{ Direction } & \multicolumn{2}{|c|}{ Delay/s } \\
\hline A & B & A & B & short & long \\
\hline Slow & moderate & $\begin{array}{l}\text { horizontal } \\
\text { oblique } \\
\text { horizontal } \\
\text { oblique }\end{array}$ & $\begin{array}{l}\text { horizontal } \\
\text { oblique } \\
\text { horizontal } \\
\text { oblique } \\
\text { horizontal } \\
\text { oblique } \\
\text { horizontal } \\
\text { oblique }\end{array}$ & $\begin{array}{l}83.75 \\
16.07 \\
19.21 \\
78.75 \\
10.43 \\
22.50 \\
12.32 \\
28.21\end{array}$ & $\begin{array}{l}75.07 \\
16.61 \\
20.82 \\
80.36 \\
18.75 \\
10.18 \\
10.71 \\
31.79\end{array}$ \\
\hline Moderate & moderate & $\begin{array}{l}\text { horizontal } \\
\text { oblique } \\
\text { horizontal } \\
\text { oblique }\end{array}$ & $\begin{array}{l}\text { horizontal } \\
\text { oblique } \\
\text { horizontal } \\
\text { oblique } \\
\text { horizontal } \\
\text { oblique } \\
\text { horizontal } \\
\text { oblique }\end{array}$ & $\begin{array}{l}45.71 \\
18.50 \\
26.50 \\
42.07 \\
78.04 \\
31.79 \\
29.46 \\
84.46\end{array}$ & $\begin{array}{r}38.93 \\
9.89 \\
22.21 \\
43.32 \\
73.04 \\
17.32 \\
27.14 \\
75.11\end{array}$ \\
\hline
\end{tabular}


There was a significant two-way interaction between speed of $\mathrm{A}$ and speed of $\mathrm{B}$ $(F=155.19, p<0.001)$, with higher ratings when the two speeds were the same than when they were different. There was a significant two-way interaction between direction of $\mathrm{A}$ and direction of $\mathrm{B}(F=127.76, p<0.001)$, with higher ratings when the two directions were the same than when they were different. These were both qualified by a significant four-way interaction between speed of $\mathrm{A}$, speed of $\mathrm{B}$, direction of $\mathrm{A}$, and direction of $\mathrm{B}(F=74.74, p<0.001)$, and it is this interaction that most clearly reveals the main finding.

The interaction is depicted in figure 3. In the key to this figure the variables are identified, in order, as speed of object A, slow (S) or moderate (M), speed of object B, slow $(\mathrm{S})$ or moderate $(\mathrm{M})$, and direction of object $\mathrm{A}$, horizontal $(\mathrm{H})$ or oblique $(\mathrm{O})$. Means were high (ranging from 75.54 to 79.79) whenever objects A and B shared the same speed and direction: that is, for SSH and $\mathrm{MMH}$ when the direction of object $\mathrm{B}$ was horizontal, and for SSO and MMO when the direction of object B was oblique. In all other combinations means were low, ranging from 11.52 to 42.70 . This finding supports the property-transmission hypothesis. There was no significant main effect or interaction with delay.

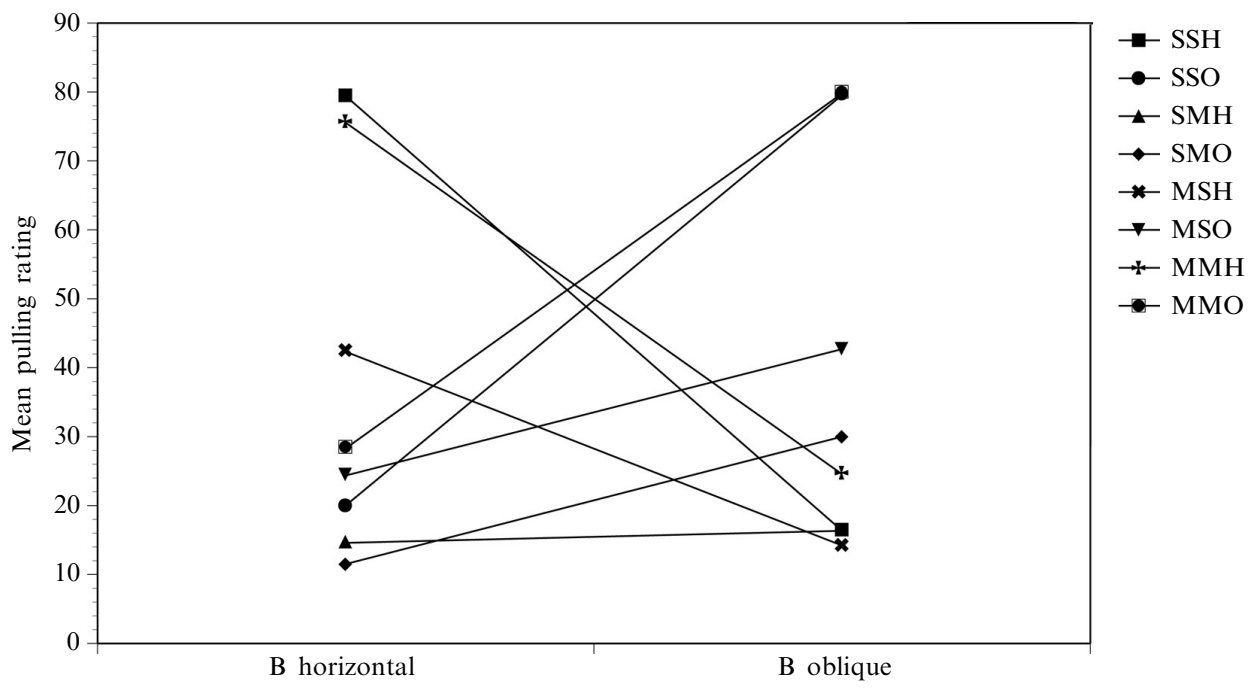

Figure 3. Four-way interaction between speed of object $A$, speed of object $B$, direction of $A$, and direction of $\mathrm{B}$. The key identifies levels of variables, respectively: speed of object A (slow, S, versus moderate, M); speed of object B (slow, S, versus long, M); direction of object A (horizontal, $\mathrm{H}$, versus oblique, $\mathrm{O})$.

Figure 3 indicates that there is more to the four-way interaction than just the effect described in the previous paragraph. Some light can be shed on this by considering degrees of resemblance between the kinematic properties of the objects within stimuli. The objects can match in either speed or direction of motion, so each stimulus can be categorised as matching on both properties, on one, or on none. Mean pulling ratings were 78.57 for stimuli matching on both properties, 27.35 for stimuli matching on just one, and 16.60 for stimuli matching on none. The two stimuli with means just over 40 in figure 3 both match on one property (direction in both cases). So, even when the reported pulling impression is weak, it appears to be less weak for stimuli that have partial resemblance in kinematic properties than for stimuli that have none. This is not a complete account of the results, however, and analysis of other significant results sheds more light on the trends in the ratings. 


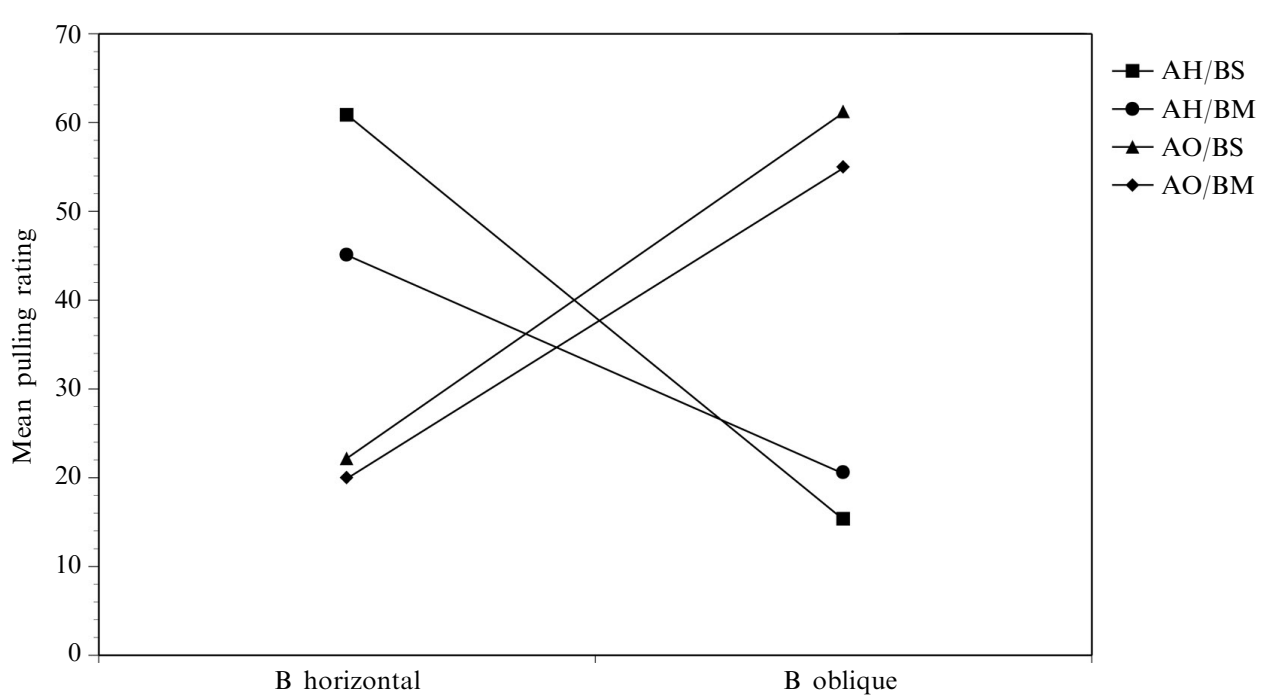

Figure 4. Three-way interaction between speed of object B, direction of object A, and direction of object B. The key identifies levels of variables, respectively; object A, horizontal $(\mathrm{H})$ or oblique $(\mathrm{O})$, and object $\mathrm{B}$, slow $(\mathrm{S})$ and moderate $(\mathrm{M})$.

There was a significant interaction between speed of object $\mathrm{B}$ and direction of object $\mathrm{B}(F=14.25, \mathrm{MSE}=284.75, p<0.001)$. This was qualified by a three-way interaction between speed of $\mathrm{B}$, direction of $\mathrm{B}$, and direction of $\mathrm{A}(F=25.78, \mathrm{MSE}=337.68$, $p<0.001)$. This is depicted in figure 4. In the key to this figure, motion of object $\mathrm{A}$ is identified as horizontal $(\mathrm{H})$ or oblique $(\mathrm{O})$, and speed of object $\mathrm{B}$ as slow $(\mathrm{S})$ or moderate (M). The main feature of the interaction is that, when both objects are moving horizontally, pulling ratings were higher when object B was moving at slow speed than at moderate speed. This tendency is weaker or absent when both objects are moving obliquely, and absent or reversed when object A is moving horizontally and object B obliquely. This interaction is, of course, further qualified by the four-way interaction shown in figure 3. The key points of relevance in that figure are the two means that are just above 40 . These are the two highest means for any stimuli in which the two objects do not share both speed and direction properties. In both of them object A moves at moderate speed and object B slowly, so that object B gradually trails further behind object $\mathrm{A}$. It is not clear why this combination of features should give rise to higher pulling ratings than others in which the objects do not share all their kinematic properties. Possibly stimuli of this kind remind participants (or their perceptual systems) of pulling events in which the connection between the objects is elastic, but such events are not particularly common. This feature of the results therefore remains in need of explanation.

The one remaining significant result was a main effect of speed of object A $(F=21.48, \mathrm{MSE}=666.98, p<0.001)$, with a higher mean at moderate speed (41.47) than at slow speed (33.47). This result is explained by the same feature of the four-way interaction: of the stimuli where the two objects did not share all kinematic properties, means for two were higher than the remainder, as shown in figure 3, and in both of these object A was moving at moderate speed and object B at slow speed. The main effect of object B was not significant by the level set for this analysis, but would have been significant at the 0.05 level $(F=7.18, \mathrm{MSE}=713.36, p<0.05)$. This shows the difference that would be predicted from the foregoing results, namely a higher mean at slow speed (39.86) than at moderate speed (35.08). 


\section{Discussion}

As figure 1 shows, the two objects have no visible connection, never come into contact, and never approach each other. The stimulus sequence is highly ambiguous and is compatible with several possible physical interpretations. For example, both A and B might have been made to move by unseen objects striking them. The objects could also be moving independently, in other words with no interaction taking place between them. Yet stimuli in which speed and direction were the same for both objects reliably gave rise to an impression that object $\mathrm{A}$ was pulling object $\mathrm{B}$, an impression qualitatively similar to that reported by White and Milne (1997) in the case of five-object stimuli. Manipulating delay had no significant effect on this. The pulling impression was substantially weakened if the motions of the object differed in either speed or direction, and weakened further if the objects differed in both respects. These results are consistent with the property-transmission hypothesis.

The property-transmission hypothesis does not stipulate that the properties of the two objects must match in every respect for an impression of pulling to occur. The minimum requirement is appropriately time-ordered resemblance in at least one salient property of cause and effect when the other two conditions listed in the introduction are met. Matching in one respect is better than matching in none. So the tendency for means to be higher when the objects shared either similar speed or similar direction than when they shared neither is also consistent with the property-transmission hypothesis. Nevertheless, mean ratings were consistently high when the kinematic properties of the objects matched in both speed and direction, and consistently low when they did not. The highest mean for any stimulus where either speed or direction were not identical was 45.71, and the lowest for any stimulus where both speed and direction were identical was 73.04. So it appears that complete resemblance in kinematic properties counts for more than resemblance in one out of two.

It could be argued that the differences in speed and direction used in this research were substantial. The difference in speed was $2.7 \mathrm{~cm} \mathrm{~s}^{-1}$. Although the absolute difference in speeds was not great, one of them was $50 \%$ faster than the other. The difference in direction was $30^{\circ}$. It may be that smaller differences in speed or direction would result in higher ratings of pulling. There is evidence that observers have poor sensitivity to changes in speed (Runeson 1974, 1975), which could indicate that small differences in speed could result in strong impressions of pulling because the difference in speed would not be noticed. However, there is a difference between observing a single object in motion against a background, as in Runeson's research, and observing two objects in motion at the same time. Differences in speed in the latter case could be readily inferred from changes in spatial separation of the objects, which might be easier to pick up. The present results provide grounds for claiming that the pulling impression is maximal with exact identity of kinematic properties, but the degree of sensitivity of the effect to variations in relative speed and direction has yet to be ascertained.

It could also be argued that the results are not surprising because exact similarity of speed and direction are characteristic of pulling relations between real objects. Trains pulling carriages and lorries pulling trailers are obvious examples. However, in the stimuli used in this research there are substantial gaps between the objects when both are in motion, as illustrated in figure 1. Such gaps are more characteristic of objects linked by longer connections, including chains and string. In such cases exact similarity of speed and direction are not inevitable, nor even necessarily common. Consider one car pulling another by means of a rope stretched between them. Exact similarity of direction will occur only when the cars are moving in a straight line. When the lead car rounds a bend, the car being pulled will be moving in a different direction until it has rounded the bend and is once more in line astern. Yet pulling 
continues throughout the cornering manoeuvre. A water skier is towed by a speedboat, but deliberately moves off to the side of the speedboat's path for the purpose of manoeuvres such as slalom. A kite towed by a person running on the ground may exhibit many and rapid changes in direction, and yet is still being pulled by the person holding the string. The kite also illustrates the point that divergence of direction also permits difference in speed. Pulling only occurs when the connection is taut, so pulling in a straight line implies constant speed unless the connection is noticeably elastic, which is not usual. But changes in direction of a kite's motion imply that it must be moving appreciably faster than the person pulling it, even though the connection remains taut. The size of the speed difference is related to the size of the difference in directions of motion. Stimuli in the present experiment in which object A moved horizontally and object B moved obliquely constitute a simplified representation of the kinematics of a kite being lifted off the ground by a running person. Yet these stimuli gave rise to weak pulling impressions or none at all. Variations in strength of the pulling impression therefore relate more closely to the occurrence or non-occurrence of property transmission than to variations in the features of real pulling events.

Against this, it could be argued that the stimuli are presented in a featureless context. Under this circumstance, perception could be guided by the most parsimonious physical assumptions, which would essentially be the Newtonian assumption of motion in a straight line in the absence of external forces. Under such an assumption, matching of kinematic properties would be expected because there are no physical grounds for expecting anything else. This argument has two main flaws. One is that perception is not guided by Newtonian assumptions. Numerous studies have shown that motion is perceived in accordance with non-Newtonian assumptions such as impetus theory, or in accordance with assumptions about the physical environment in which it is occurring, such as the presence of a gravitational field (Hubbard 1995, 2005; Kozhevnikov and Hegarty 2001; McCloskey 1983). Under those assumptions objects are not necessarily expected or perceived to move in straight lines or at constant speed. The second flaw is that making the most parsimonious assumptions does not explain why an impression of pulling occurs at all. There is no visible connection between the objects, and inferring an unseen connection would be unparsimonious. Object B should be perceived as moving autonomously, on the grounds that there is no visible cause of its motion. Perception is undoubtedly guided by assumptions about the likely physical context of motion, but those assumptions are neither Newtonian nor parsimonious, and result in perceptual interpretations that go well beyond what the available information supplies.

Michotte (1963) treated the traction effect (his term for the visual impression of pulling) under his theoretical account of entraining. Michotte's explanation for the launching and entraining effects refers to a perceptual structure called the ampliation of the movement. According to this, the generation of object B's movement is understood simply as the displacement or phenomenal extension of object A's movement onto object B. Michotte (1963) defined ampliation as "the creating or establishing of the extension on to a second object of the already existing movement of a first object, in such a way that this movement brings about the displacement of the second object" (page 143, italics in original).

The only feature of the stimuli that matters for the entraining effect to occur is the continuation of object A's prior motion in both objects: that is, the entraining effect is a matter of motion characteristics and nothing else. This idea conforms to the Gestalt principle of common fate (Michotte and Thinès 1991). Conditions that disrupt the common fate of the objects are associated with weakening or absence of the entraining impression. Michotte argued that common fate was also a feature of stimuli that gave rise to the pulling impression. For example, in one study, "object A, moving from left to right, joined $\mathrm{B}$ and passed beyond it; and when it was $5 \mathrm{~mm}$ away $\mathrm{B}$ began to move 
in its turn in the same direction and at the same speed". Michotte stated that $58 \%$ of a sample of observers reported the traction effect with this stimulus. The objects do not have to be in contact, so long as the conditions for common fate are satisfied.

This appears to indicate that Michotte's account of the entraining effect in application to the pulling impression makes the same predictions as the property-transmission hypothesis: if the objects have the same kinematic properties, they have common fate, and the pulling impression occurs; otherwise, not. But, in fact, Michotte's account does not hold for the stimuli used in the present experiment. Michotte's description of the first phase of motion in the entraining effect is clear: "there are two objects, one of which moves in the direction of the other and joins it" (1963, page 317). The stimulus presented in the experiment described in the previous paragraph exemplifies this. In fact, this prior approach phase is necessary for the ampliation of the movement to occur: in ampliation, object A's movement is extended onto object $\mathrm{B}$, and this cannot happen if object A only ever moves away from object B. But, in the present study, object A did not move towards object B. As soon as object A began to move, its motion took it away from object B. Under this condition, there is no possibility that object A's motion could be displaced onto object B, and Michotte's explanation cannot account for the finding that pulling impressions occurred with this stimulus.

The property-transmission hypothesis does not require that object A must move towards object B: so long as there is sufficient spatial proximity for the two objects to be integrated into a single percept, similarity of speed and direction are sufficient. The role of causal object is assigned on the basis of priority of motion: object $\mathrm{A}$ is perceived as causal because it moves first, not because its motion takes it toward object B. If the objects start to move at the same time, there is no impression of pulling (White and Milne 1997). So the present results favour the property-transmission hypothesis over Michotte's common-fate account.

In the introduction, it was stated that three criteria serve to identify perceived or inferred property transmission. Of these, the first, activity going on, is satisfied by the motion of the objects, and more particularly the prior motion of object A, which may be important to the assignment of causal roles to the two objects. The third, salient timeordered resemblance between the properties of causal object and effect object, is satisfied by similarity of speed and direction of motion, the stimulus conditions that gave rise to the strongest impressions of pulling. The second, presence of cues to generative transmission, is more problematic. The objects move in a featureless space, with no visible connection between them or transmission of anything from one to the other. It may be that the generative-transmission-cues condition is a requirement for inference of causality via property transmission but not for a visual causal impression of causality. Alternatively, it may be that the satisfaction of this condition is an assumption that is made because of the satisfaction of the other two conditions. Although no connection between the objects is visible, the presence of an unseen connection may form part of the perceptual interpretation of the stimulus if the other features of it favour a pulling interpretation. The role of generative transmission cues could well depend on the kind of physical system in question: such cues may be important in the kinds of causal systems investigated by Shultz (1982), which concerned spatially separate causes and effects such as bellows and a candle flame, a tuning fork and a resonating box, and a flashlight and a Crookes' radiometer, but less so in other contexts. I would tentatively suggest that spatial proximity matters, but the relation between this and generative relations cues is a matter that would greatly benefit from further research.

The key point of this research, then, is that causal impressions of launching, entraining, and pulling are maximised when the conditions for property transmission are met, and that this does not accurately represent what happens in real causal relations, be they collisions between billiard balls or people pulling kites. Differences in 
speed and direction of motion between object A and object B are common occurrences in real causal relations, so it is necessary to ask why stimuli representing such differences tend not to give rise to causal impressions. The property-transmission hypothesis provides an answer to that question.

Property transmission is a domain-general hypothesis about causality, and evidence for it can be found in explicit reasoning about causal relations as well as implicit reasoning and perception (White 2009). I argued that it is adopted as a general hypothesis about causality, and that it influences causal inferences made under conditions of uncertainty, where it tends to be applied beyond its proper bounds. The present research exemplifies this: there is uncertainty because the pulling stimulus is ambiguous and compatible with different physical interpretations. In real-world cases of pulling the stimulus information is likely to be less ambiguous, in the sense that cues to generative relations such as physical connections between the objects are more likely to be perceived. The idea that property transmission is a guide to causality in conditions of uncertainty is also exemplified by phenomena of judgment such as some kinds of illusory correlation (Chapman and Chapman 1967; King and Koehler 2000) and belief in magical contagion (Nemeroff 1995; Nemeroff and Rozin 1994; White 2009). Phenomenal causality has always been considered a thing apart from other forms of judged and inferred causality. The present research is an indication that there may, after all, be connections between them, and that a general heuristic for inferring causality may apply both in visual perception and in higher cognition.

Acknowledgments. Thanks to Sophie Patterson for acting as experimenter and to Lorraine Woods for assistance with the figures. The software used to create the stimuli was designed by Alan Milne.

\section{References}

Beasley N E, 1968 "The extent of individual differences in the perception of causality" Canadian Journal of Psychology 22 399-407

Chapman L J, Chapman J P, 1967 "Genesis of popular but erroneous diagnostic observations" Journal of Abnormal Psychology $72193-204$

Choi H, Scholl B J, 2004 "Effects of grouping and attention on perception of causality" Perception \& Psychophysics $66926-942$

Choi H, Scholl B J, 2006 "Perceiving causality after the fact: postdiction in the temporal dynamics of causal perception" Perception 35 385-399

Gordon I E, Day R H, Stecher E J, 1990 "Perceived causality occurs with stroboscopic movement of one or both stimulus elements" Perception $1917-20$

Hubbard T L, 1995 "Cognitive representation of motion: evidence for friction and gravity analogues" Journal of Experimental Psychology: Learning, Memory, and Cognition 21241 - 254

Hubbard T L, 2005 "Representational momentum and related displacements in spatial memory: a review of the findings" Psychonomic Bulletin \& Review $12822-851$

King R N, Koehler D J, 2000 "Illusory correlations in graphological inference" Journal of Experimental Psychology: Applied $6336-348$

Kozhevnikov M, Hegarty M, 2001 "Impetus beliefs as default heuristics: dissociation between explicit and implicit knowledge about motion" Psychonomic Bulletin \& Review $8439-453$

Leslie A M, Keeble S, 1987 "Do six-month-old infants perceive causality?" Cognition 25 265-288

McCloskey M, 1983 "Intuitive physics" Scientific American $248395-400$

Michotte A, 1946 La Perception de la Causalité (Louvain: Institut Supérieur de Philosophie)

Michotte A, 1963 The Perception of Causality (New York: Basic Books)

Michotte A, Thinès G, 1991 "Perceived causality", in Michotte's Experimental Phenomenology of Perception Eds G Thinès, A Costall, G Butterworth (Hove, East Sussex: Lawrence Erlbaum Associates) pp $66-87$

Natsoulas T, 1961 "Principles of momentum and kinetic energy in the perception of causality" American Journal of Psychology $74394-402$

Nemeroff C, 1995 "Magical thinking about illness virulence: conceptions of germs from 'safe' versus 'dangerous' others" Health Psychology 14 147-151

Nemeroff C, Rozin P, 1994 "The contagion concept in adult thinking in the United States: transmission of germs and of interpersonal influence" Ethos 22 158-186 
Newman G E, Choi H, Wynn K, Scholl B J, 2008 "The origins of causal perception: evidence from postdictive processing in infancy" Cognitive Psychology $57262-291$

Runeson S, 1974 "Constant velocity - not perceived as such" Psychological Research 37 3-23

Runeson S, 1975 "Visual prediction of collision with natural and nonnatural motion functions" Perception \& Psychophysics $18261-266$

Saxe R, Carey S, 2006 “The perception of causality in infancy" Acta Psychologica 123 144-165

Schlottmann A, Ray E, Mitchell A, Demetriou N, 2006 "Perceived social and physical causality in animated motions: spontaneous reports and ratings" Acta Psychologica $123112-143$

Scholl B J, Tremoulet P D, 2000 "Perceptual causality and animacy" Trends in Cognitive Sciences $4299-309$

Shultz T R, 1982 "Rules of causal attribution" Monographs of the Society for Research in Child Development 47 1-51

White P A, 2006 "The causal asymmetry" Psychological Review $113132-147$

White P A, 2009 "Property transmission: an explanatory account of the role of similarity information in causal inference" Psychological Bulletin $135774-793$

White P A, Milne A, 1997 "Phenomenal causality: impressions of pulling in the visual perception of objects in motion" American Journal of Psychology 110 573-602

White P A, Milne A, 1999 "Impressions of enforced disintegration and bursting in the visual perception of collision events" Journal of Experimental Psychology: General 128 499-516

White P A, Milne A, 2003 "Visual impressions of penetration in the perception of objects in motion" Visual Cognition $10605-619$ 


\section{PERTEPTION}

VOLUME 392010

www.perceptionweb.com

Conditions of use. This article may be downloaded from the Perception website for personal research by members of subscribing organisations. Authors are entitled to distribute their own article (in printed form or by e-mail) to up to 50 people. This PDF may not be placed on any website (or other online distribution system) without permission of the publisher. 